\title{
Effect of Surface Active Ions on the Rate of Water Evaporation
}

\author{
Walter S. Drisdell, Richard J. Saykally,* and Ronald C. Cohen* \\ Department of Chemistry, UC Berkeley, Berkeley, California 94720, and Department of Earth and Planetary \\ Science, UC Berkeley, Berkeley, California 94720
}

Received: February 25, 2010; Revised Manuscript Received: May 3, 2010

\begin{abstract}
Current understanding of the vapor-liquid exchange kinetics of liquid water is incomplete, leading to uncertainties in modeling the climatic effects of clouds and aerosol. Initial studies of atmospherically relevant solutes (ammonium sulfate, sodium chloride) indicate that their effect on the evaporation kinetics of water is minimal, but all those constituent ions are also expected to be depleted in concentration at the air-water interface. We present measurements of the evaporation kinetics of water from $4 \mathrm{M}$ sodium perchlorate solution, which is expected to have an enhanced concentration of perchlorate in the surface layer, using Raman thermometry of liquid microdroplets in a free evaporation regime. We determine the evaporation coefficient $\gamma_{\mathrm{e}}$ to be $0.47 \pm 0.02$, ca. $25 \%$ smaller than our measured value for pure water $(0.62 \pm 0.09)$. This change, while small, indicates that direct interactions between perchlorate ions and evaporating water molecules are affecting the evaporation mechanism and kinetics and suggests that other solutes with high surface affinities may also produce a similar influence in the atmosphere and elsewhere.
\end{abstract}

\section{Introduction}

The vapor-liquid exchange of water is of interest in many areas of science, and particularly in atmospheric chemistry, since the kinetics and equlibria strongly influence aerosol and cloud properties and thus terrestrial climate. Nevertheless, current understanding of the fundamental processes involved is poor. In the atmosphere, the interactions between clouds and aerosol particles and their effects on climate remain the largest uncertainties climate modeling, due in part to our inability to accurately quantify the microscopic liquid-vapor exchange rates on ambient particles. ${ }^{1}$ The problem is compounded by the presence of dissolved solutes as well as organic surfactant species that are prevalent in atmospheric aerosol, but even attempts to quantify vapor-liquid exchange kinetics for pure water have proven difficult. Previous attempts to measure these rates have yielded values that span 3 orders of magnitude, but recent measurements have converged to within a single order of magnitude. ${ }^{2-10}$ Several factors have contributed to experimental difficulties, including accounting for simultaneous evaporation and condensation, accurately determining the liquid surface temperature during the mass transfer process, and quantifying the complex thermal and mass fluxes in the vapor phase. ${ }^{5,7}$ Some recent studies have suggested that the changing position of the liquid-vapor boundary on the time scale of the experiments can cause problems, especially when using a computer model to interpret experimental data. ${ }^{9,11}$

In our own previous studies, we have employed liquid microjets coupled with Raman thermometry to examine water evaporation without accompanying condensation., ${ }^{2,3}$ This technique simplifies the interpretation of the data significantly, allowing the evaporation rate to be derived from the data using a simple evaporative cooling model. For both pure $\mathrm{H}_{2} \mathrm{O}$ and pure $\mathrm{D}_{2} \mathrm{O}$ we found that the evaporation process proceeds at $\sim 60 \%$ of the thermodynamic maximum rate derived from

* To whom correspondence should be addressed: Tel (510) 642-8269, e-mailsaykally@berkeley.edu (R.J.S.); Tel (510) 642-2735, e-mail rccohen@berkeley.edu (R.C.C.). kinetic gas theory. This is not slow enough to affect cloud growth rates in current models (which assume the maximum rate). ${ }^{12}$ There are, however, several field studies which suggest that aerosol and cloud droplet growth rates in the atmosphere are often much slower than this, likely due to the presence of dissolved impurities and organic surfactant coatings on evaporating particles. ${ }^{12-14}$ To determine the effects of such species, we have recently performed similar evaporation measurements on $3 \mathrm{M}$ ammonium sulfate solution. ${ }^{15}$ Ammonium sulfate was selected because it represents the largest inorganic component of anthropogenic aerosol. ${ }^{16} \mathrm{We}$ found that, while the thermodynamic maximum evaporation rate for $3 \mathrm{M}$ ammonium sulfate solution is lower than that of pure water by $\sim 13 \%$, water still evaporated from the solution at ca. $60 \%$ of this maximum rate. The lack of change in the kinetics is consistent with calculations and experiments showing that both the ammonium and sulfate ions are depleted from the interfacial layer and therefore have limited interactions directly with evaporating water molecules at the surface. ${ }^{15,17,18}$ Other atmospherically relevant ionic solutes, such as sodium, chloride, and nitrate, are also predicted to be surface-depleted, so it seems unlikely that inorganic solutes affect liquid-vapor exchange in the atmosphere. ${ }^{18-22}$ It is possible, however, that ions that are enhanced in concentration at the air-water interface could have an effect on evaporation kinetics. While most of these types of ions are not atmospherically relevant, a study of their effect on the evaporation kinetics of liquid water could elucidate the underlying molecular mechanism. It is with this aim that we extend our evaporation studies to sodium perchlorate solution. Perchlorate was selected for study as it is predicted to be among the most strongly surface-enhanced ions in liquid water. ${ }^{18,23}$

In studies of evaporation and condensation, a convenient parameter to report is the evaporation coefficient or mass accommodation coefficient. This parameter is defined via the Hertz-Knudsen equation, which represents the maximum condensation rate of a gas onto a surface from kinetic gas theory: ${ }^{4}$ 


$$
J_{\mathrm{c}}=\gamma_{\mathrm{e}} \frac{p_{\text {sat }}}{\sqrt{2 \pi m k T}}
$$

Here, $J_{\mathrm{c}}$ is the condensation rate in molecules per unit area per time, $p_{\text {sat }}$ is the saturation vapor pressure of the liquid, $m$ is the molecular mass, $k$ is the Boltzmann constant, $T$ is the temperature, and $\gamma_{\mathrm{e}}$ is the evaporation coefficient (sometimes referred to in condensation studies as the mass accommodation coefficient or simply the accommodation coefficient, denoted as $\alpha_{m}$ ). This equation applies to equilibrium, so the evaporation and condensation rates are equal and are both described by this equation. In nonequilibrium conditions, the condensation rate will depend on the gas pressure, but the evaporation rate will not, as the activity of the liquid is constant. Therefore, eq 1 can be used to describe the evaporation rate even in nonequilibrium states. The evaporation coefficient $\gamma_{\mathrm{e}}$ is a value between zero and one and can be thought of as a transition probability, representing the fraction of evaporation or condensation attempts which succeed. For condensation, each collision with the surface is considered a condensation attempt. A coefficient of unity indicates that the process proceeds at the thermodynamic maximum rate, whereas lower values indicate that a kinetic process limits the rate. Recent measurements of this quantity have yielded values spanning the range of $0.13-1$ for pure water. $^{7-9}$ From our own measurements, we have determined the evaporation coefficient for pure $\mathrm{H}_{2} \mathrm{O}$ and pure $\mathrm{D}_{2} \mathrm{O}$ to be 0.62 \pm 0.09 ( 2 standard deviations) and $0.57 \pm 0.06$ (95\% confidence interval), respectively. ${ }^{2,3}$ Our recent study of ammonium sulfate solution showed no change, with a value of $0.58 \pm 0.05(95 \%$ confidence interval) for $\gamma_{\mathrm{e}}{ }^{15}$ In the present study, we perform similar measurements of $4 \mathrm{M}$ sodium perchlorate solution. As in our study of ammonium sulfate, the maximum thermodynamic evaporation rate for the sodium perchlorate solution as determined by eq 1 will be slower than that for pure water (by $\sim 17 \%$ ) as a result of the lower vapor pressure of the solution (see below for details of the vapor pressure estimation); the value determined for $\gamma_{\mathrm{e}}$ will therefore reflect the percentage of this lower maximum rate at which evaporation actually proceeds. A $4 \mathrm{M}$ solution was the highest concentration that did not result in clogged orifices or complications in the spectral analysis. This concentration is lower than that expected for atmospheric inorganic constituents such as ammonium sulfate, which are often supersaturated by up to twice the saturation limit. ${ }^{15}$

The high precision of our experimental technique is due to the fact that experiments are performed in the absence of condensation, allowing for the data to be interpreted with a relatively simple evaporative cooling model. We ensure that the droplets produced in our vacuum chamber are in the regime where condensation is negligible by ensuring the mean free path of evaporating molecules is large compared to the diameter of the droplets. We use the relationship between the Knudsen number and the number of collisions experienced by an evaporating molecule moving from the surface of the droplet to an infinite distance to estimate the required droplet size:

$$
\begin{array}{r}
N_{\mathrm{coll}}\left(r_{0}, T\right)=\int_{r_{0}}^{\infty} \frac{\mathrm{d} r}{\lambda(r, T)}=\sqrt{2} \pi d_{\mathrm{coll}}{ }^{2} n\left(r_{0}\right) r_{0}{ }^{2} \int_{r_{0}}^{\infty} \frac{\mathrm{d} r}{r^{2}}= \\
\frac{r_{0}}{\lambda\left(r_{0}, T\right)}=\frac{1}{K n\left(r_{0}, T\right)}
\end{array}
$$

In this expression, $N_{\text {coll }}(r, T)$ is the number of collisions experienced by an evaporating droplet starting at distance $r$ from the droplet center, $\lambda(r, T)=\left[\sqrt{ } 2 \pi d_{\text {coll }}^{2} n(r)\right]^{-1}$ is the mean free path in the vapor phase, $r_{0}$ is the radius of the droplet, $d_{\text {coll }}$ is the collision diameter of $\mathrm{H}_{2} \mathrm{O}, n(r)$ is the number density of the vapor, and $K n=\lambda / r$ is the Knudsen number. For $K n>1$, evaporating molecules undergo less than one collision on average, ensuring that none return to the droplet and condense. Droplets used in this study were between 9.15 and $12.3 \mu \mathrm{m}$ in radius; the mean free path of evaporate from the $4 \mathrm{M}$ sodium perchlorate solution reaches $12.3 \mu \mathrm{m}$ at temperatures of $283 \mathrm{~K}$ and colder. Only a small portion of the data taken in this study was above this temperature (up to $285.5 \mathrm{~K}$, typically only one measured temperature point out of $20-25$ points per experimental run). Given that our data do not show any dependence on droplet size and that our model fits are unchanged when ignoring any temperature points above $283 \mathrm{~K}$, we believe that any effects from gas-phase collisions in the first $\sim 75 \mu \mathrm{s}$ of measurement are smaller than our experimental precision. Even if vapor-phase collisions occurred in these cases, solid angle considerations imply that only a fraction of the colliding molecules would actually impinge upon the droplet. Additionally, we have seen no evidence for condensation effects in our previous studies for droplet sizes smaller than $20 \mu$ m radius. ${ }^{2,3,15}$

\section{Experimental Section}

Sample Preparation. Anhydrous sodium perchlorate was obtained commercially (Sigma, ACS reagent, $\geq 98 \%$ ). Solutions were prepared volumetrically using filtered and deionized $\mathrm{H}_{2} \mathrm{O}$ (18.2 M $\Omega$ resistivity, Milli-Q, Millipore). Solutions were additionally filtered through disposable $0.5 \mu \mathrm{m}$ particle retention filters and stored in a sealed container when not in use.

Experimental Apparatus. The experimental apparatus has been described in detail previously. ${ }^{3,15}$ Briefly, the liquid orifice is mounted on a piezoelectric ceramic which acts as a vibrating orifice aerosol generator (VOAG). This allows the droplets produced by the jet to be forced into a uniform size by applying a square-wave driving frequency from a function generator. Orifices used in this study ranged between 4.3 and $6.6 \mu \mathrm{m}$ radius and were produced from $100 \mu \mathrm{m}$ i.d. fused silica tubing using a commercial $\mathrm{CO}_{2}$ laser pipet puller (Sutter Instrument Model P2000). Orifices were sized using angle-resolved Mie scattering as reported previously. ${ }^{3,10}$ The VOAG apparatus is, in turn, mounted on an $X Y Z$ manipulator and attached via bellows to the $7 \mathrm{~cm}$ cubical vacuum chamber, with the droplet stream propagating downward. The vacuum chamber is pumped by a $110 \mathrm{~L} / \mathrm{s}$ turbomolecular pump. The droplet stream is intersected by the beam of an $\mathrm{Ar}^{+}$laser operating at $\sim 250 \mathrm{~mW}$ or less at $514.5 \mathrm{~nm}$. Raman scatter is collected at $90^{\circ}$, filtered, focused, and sent via optical fiber to a monochromator $(f / 6.5)$ equipped with a liquid nitrogen-cooled CCD detector. After the laser light has intersected the droplet train, it is collected by a photodiode and sent to an oscilloscope. This allows real-time monitoring of the droplets produced by the VOAG; as droplets pass through the laser focus, there is a dip in signal on the photodiode. A sinusoidal signal on the oscilloscope indicates the presence of uniformly sized droplets. After the droplets have passed through the interaction region, they enter a liquid nitrogen-cooled trap to freeze the liquid and prevent any re-evaporation.

The OH stretching region of the Raman spectrum (2500-3900 $\mathrm{cm}^{-1}$ ) is used to determine the temperature of the liquid to within $\pm 2 \mathrm{~K}$. Calibration spectra of the $4 \mathrm{M}$ solution were taken in a cuvette at temperatures between $\sim 273$ and $\sim 325 \mathrm{~K}$ monitored with a thermocouple. Example calibration spectra are shown in Figure 1a and show a feature at $\sim 3580 \mathrm{~cm}^{-1}$ due to the perchlorate ion. ${ }^{24}$ This feature partially obscures the $\mathrm{H}_{2} \mathrm{O}$ 

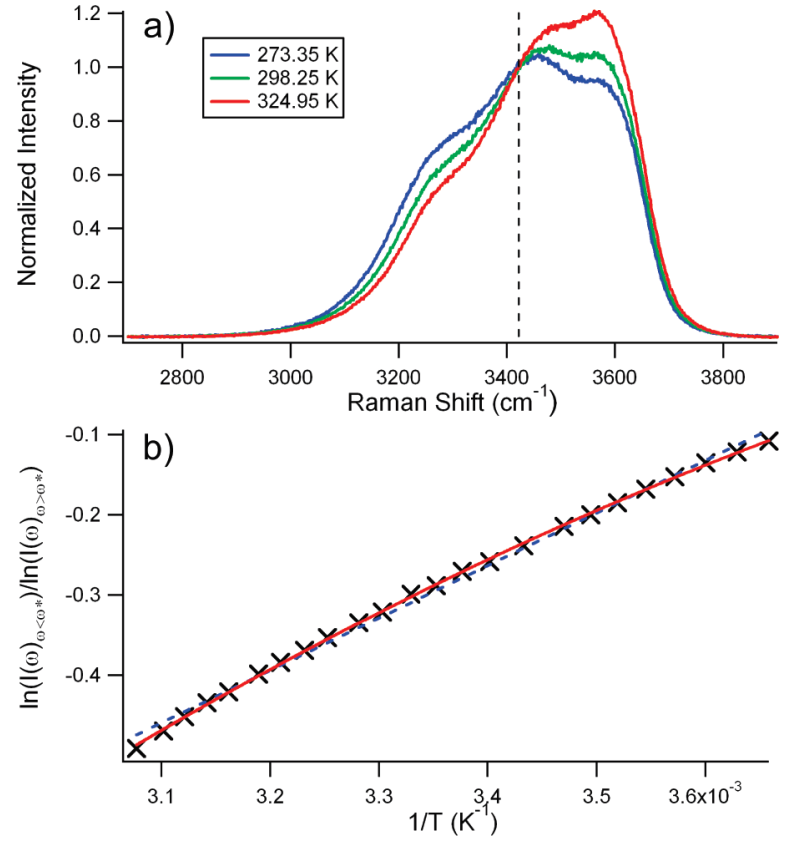

Figure 1. (a) Representative spectra used to generate a temperature calibration curve. The spectral feature at $\sim 3580 \mathrm{~cm}^{-1}$ is due to the perchlorate anion. The full calibration curve was constructed from 24 spectra taken at varying temperatures between 273.35 and $324.95 \mathrm{~K}$ (both extremes are shown). The dashed line indicates the frequency $\omega^{*}=3422.6 \mathrm{~cm}^{-1}$ where the spectra were split in order to construct the calibration curve. (b) The calibration curve constructed from the spectra. The red line shows the quadratic fit used for the calibration curve. The dashed blue line shows a linear fit, for contrast. The quadratic fit was used due to better self-reproduction of the calibration temperatures.

spectrum and causes slightly lower precision in the temperature derivation relative to pure water, while still being within \pm 2 K. Spectra were split at $3422.6 \mathrm{~cm}^{-1}$, and the ratio of the area under the spectrum for frequencies below this point to the area under the spectrum for frequencies above this point was plotted against $1 / T$ to create a temperature calibration curve. In our previous studies, the resulting curve was linear, ${ }^{2,3,15}$ but in this study we observed a slight curvature and found a quadratic fit to reproduce the calibration data more accurately, especially the lower temperatures. Figure $1 \mathrm{~b}$ shows a sample calibration curve with both linear and quadratic fits.

In our study of ammonium sulfate, we predicted a $\sim 5 \%$ concentration increase to occur during experiments as a result of evaporative loss of water from the solution. ${ }^{15}$ While this small change was not expected to affect the evaporation kinetics, we found that the Raman spectral shape was quite sensitive to concentration changes and that our calibrations needed to account for the change to avoid skewing our derived temperatures. Specifically, we took a second temperature calibration at $6 \%$ higher salt concentration and interpolated between the two calibrations based on the expected concentration increase over time. To determine if the same correction is necessary for sodium perchlorate solutions, we used a 4.24 M solution for a test calibration but found that the spectral shape was relatively insensitive to the concentration change. Corrections to the derived temperatures were less than $0.1 \mathrm{~K}$, much smaller than the precision of the temperature derivation. Therefore, we simply used $4 \mathrm{M}$ calibration curves for all data.

Evaporative Cooling Model. The discrete evaporative cooling model used to interpret our temperature data has been described in detail previously. ${ }^{2,3,15}$ The model divides the droplet into 20 concentric spherical shells, each with its own temperature. The calculation is performed in two steps; in the first the outermost shell is allowed to evaporate and therefore cool, and in the second step heat is propagated outward from the inner shells according to the thermal diffusion equation. This allows an accurate representation of the surface temperature of the droplet. Mass loss from the evaporation process is also taken into account, and all shells are resized accordingly. The process is then iterated. We determine $\gamma_{\mathrm{e}}$ by matching the predicted cooling rate from the model to that observed in our experiments. The cooling rate of outermost shell of the modeled droplet can be expressed as

$$
\frac{\mathrm{d} T}{\mathrm{~d} t}=\gamma_{\mathrm{e}} \frac{p_{\text {sat }}}{\sqrt{2 \pi m k T}} \frac{\Delta H_{\mathrm{vap}}}{C_{p}} \frac{3 r_{0}{ }^{2}}{\left(r_{0}{ }^{3}-r_{1}{ }^{3}\right) \rho}
$$

where $p_{\text {sat }}$ is the saturation vapor pressure, $m$ is the mass of an evaporating molecule, $k$ is the Boltzmann constant, $T$ is the temperature in kelvin, $\Delta H_{\text {vap }}$ is the enthalpy of vaporization, $C_{p}$ is the specific heat capacity of the solution, $r_{0}$ and $r_{1}$ are the outer and inner radii of the outermost shell, respectively, and $\rho$ is the density of the solution. ${ }^{15}$ The saturation vapor pressure of the $4 \mathrm{M}$ sodium perchlorate solution was determined using the empirical temperature-dependent equation for the vapor pressure of pure water presented by Murphy and Koop ${ }^{25}$ and applying a water activity of 0.8308 as reported by Djogic and Branica (estimated to be accurate to \pm 0.001 ). ${ }^{26}$ The water activity was assumed to be constant with temperature; i.e., the temperature dependence of the vapor pressure was assumed to be of the same form as that for pure water in the formula of Murphy and Koop. While there are no temperature-dependent data on water activity for sodium perchlorate solutions, studies of a variety of other solutes over a wide concentration range indicate no measurable temperature dependence to the water activity. ${ }^{27-29}$ This in turn implies that the enthalpy of vaporization is effectively unchanged from the pure water value; therefore, we used that value $(44.4 \mathrm{~kJ} / \mathrm{mol})$ in our cooling model. The specific heat of the solution was calculated using the formula of Roth, Wolf, and Wolf. ${ }^{30}$ The density of the solution was measured to be $1.580 \mathrm{~g} / \mathrm{mL}$. Heat is then allowed to flow into the outermost shell from the shell underneath according to the thermal diffusion equation

$$
\frac{\mathrm{d} Q}{\mathrm{~d} t}=-\kappa A \frac{\mathrm{d} T}{\mathrm{~d} r}
$$

where $\mathrm{d} Q / \mathrm{d} t$ is the amount of heat transferred per time step, $\kappa$ is the thermal conductivity of the solution, $A$ is the surface area of the inner shell, and $\mathrm{d} T / \mathrm{d} r$ is the temperature difference between the two shells. A thermal conductivity of $0.5404 \mathrm{~W}$ $\mathrm{m}^{-1} \mathrm{~K}^{-1}$ was calculated for $4 \mathrm{M}$ sodium perchlorate solution using the formula of Riedel. ${ }^{31}$ The thermal diffusion process is repeated iteratively for all shells, before moving to the next time step and allowing the outermost shell to evaporate again. The volume-averaged temperature is also calculated; this is the value that is compared to experiment. In our study of ammonium sulfate, we discussed the validity of this comparison in detail; in short, the Raman response from the droplet is not uniform in space, but empirical tests of our model indicate that the volume-averaged temperature is a good approximation of the experimental data. ${ }^{15}$ The only tunable parameter in the model 


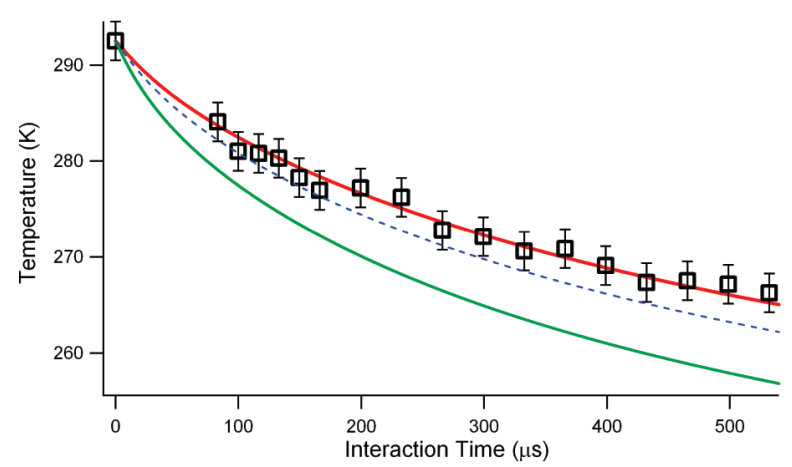

Figure 2. A representative cooling curve for $12.05 \mu \mathrm{m}$ radius droplets. The solid red line is the fit from the evaporative cooling model, yielding a $\gamma_{\mathrm{e}}$ value of 0.48 in this case. The dashed line is what the modeled cooling curve would be for $\gamma_{\mathrm{e}}=0.62$, the value we obtained previously for pure $\mathrm{H}_{2} \mathrm{O}$. The green line is the modeled cooling curve for $\gamma_{\mathrm{e}}=1$, shown for contrast.

is $\gamma_{\mathrm{e}}$, which is adjusted until the modeled temperatures match experimental values.

\section{Results}

Seven cooling curves were measured on six different droplet sizes in the range of $9.15-14.05 \mu \mathrm{m}$ radius. Unlike our previous measurements of ammonium sulfate solution, no icicles were observed to grow into the interaction region for sodium perchlorate solutions, meaning that interference from solid icicles both directly and indirectly (by providing a collision surface and enabling condensation back on the droplets) was not an issue. ${ }^{15}$ This is likely due to a difference in salt crystal structure upon freezing of the solution.

The average $\gamma_{\mathrm{e}}$ value from all data is $0.47 \pm 0.02(95 \%$ confidence interval) with a standard deviation of 0.03 . The high precision of the $95 \%$ confidence interval arises from very high consistency across data sets, despite individual temperature measurements being slightly less precise than in our previous experiments. This value of $\gamma_{\mathrm{e}}$ is lower by $\sim 25 \%$ from our value reported for pure $\mathrm{H}_{2} \mathrm{O}^{3}$ and is outside of the uncertainty range defined either as $95 \%$ confidence intervals $\left(0.62 \pm 0.03\right.$ for $\mathrm{H}_{2} \mathrm{O}$ vs $0.47 \pm 0.02$ for sodium perchlorate solution) or 2 standard deviations $\left(0.62 \pm 0.09\right.$ for $\mathrm{H}_{2} \mathrm{O}$ vs $0.47 \pm 0.06$ for sodium perchlorate solution). An example cooling curve is shown in Figure 2 for $12.05 \mu \mathrm{m}$ radius droplets, with a $\gamma_{\mathrm{e}}$ value of 0.48 . Also shown are the representative cooling curves for $\gamma_{\mathrm{e}}=0.62$, the value we measured previously for pure $\mathrm{H}_{2} \mathrm{O}$, as well as $\gamma_{\mathrm{e}}$ $=1$ for contrast. As in our previous studies, the best fit to the data arises when there is no assumed temperature dependence to $\gamma_{\mathrm{e}}{ }^{2,3,15}$

\section{Discussion}

The $\gamma_{\mathrm{e}}$ value of $0.47 \pm 0.02$ found in this study for $4 \mathrm{M}$ sodium perchlorate solution suggests that the perchlorate ion has a small but measurable effect on the evaporation kinetics of liquid water. To ensure that the observed decrease in $\gamma_{\mathrm{e}}$ is not due to incorrect parameters in our evaporative cooling model, we performed several tests of the sensitivity of the model output to the input constants. We tested a temperature-dependent value for the enthalpy of vaporization by allowing the enthalpy to increase linearly from $44.4 \mathrm{~kJ} / \mathrm{mol}$ at $295 \mathrm{~K}$ to the measured value of $46.467 \mathrm{~kJ} / \mathrm{mol}$ at $240 \mathrm{~K}$ reported by Murphy and Koop. ${ }^{25}$ This resulted in $<0.1 \%$ change in model output. Using the $3 \mathrm{M} \mathrm{NaCl}$ data of Murphy and Koop as a guide, we assumed a $5 \%$ increase in heat capacity of the solution when cooled from
295 to $240 \mathrm{~K}$, which changed the model output by $\sim 1 \%$. This change is well within the precision of our temperature derivations. We also tested temperature-dependent values for density and thermal conductivity, using the empirical formulas of Hare and Sorensen and the International Association For the Properties of Water and Steam, respectively. ${ }^{32,33}$ We modified these pure water formulas by applying our measured density and calculated thermal conductivity, but retained the form of the temperature dependence, and found $<0.1 \%$ change in model output in both cases.

We also considered whether the evaporation of the droplets might produce higher surface concentrations of the perchlorate ions, if the ions are unable to diffuse away from the interface quickly enough. This would result in a higher surface concentration in our experiments than would be expected from a $4 \mathrm{M}$ solution. A $12.05 \mu \mathrm{m}$ radius droplet is expected to shrink by $\sim 150 \mathrm{~nm}$ over $\sim 800 \mu$ s during our measurements. Using the diffusion coefficient of $1.47 \times 10^{-9} \mathrm{~m}^{2} \mathrm{~s}^{-1}$ measured for the perchlorate ion in saturated ammonium perchlorate solution by Hiquily and Clifton, ${ }^{34}$ we estimate that a perchlorate ion would diffuse $\sim 1 \mu \mathrm{m}$ within this same time period. The diffusion coefficient is expected to decrease as the droplet cools, but it is unlikely to be lowered to such an extent as to allow for concentrating of the perchlorate ions in the surface layer due to evaporation.

Another concern is whether our results could be affected by organic impurities in the salt crystals. Such impurities would likely be surface active and have been theorized lower the evaporation rate of water. ${ }^{4}$ To estimate the amount of impurities present in our solution, we performed surface tension measurements of the Millipore water used to make our solutions as well as the $4 \mathrm{M}$ solution used in the experiments, using both sessile bubble shape analysis and pendant drop shape analysis. Literature values are available for the surface tension of sodium perchlorate solutions up to $1 \mathrm{M}$ and show a linear increase of $0.22 \mathrm{mN} / \mathrm{m}$ over the pure water value. ${ }^{18,35}$ Our $4 \mathrm{M}$ solution would therefore be expected to show no more than a $0.88 \mathrm{mN} / \mathrm{m}$ increase over the pure water value, and most probably less, as the relation is not expected to remain linear for high concentrations. Using sessile bubble shape analysis, we found the surface tension of our water to be $70.24 \pm 0.1 \mathrm{mN} / \mathrm{m}$ and the surface tension of our solution to be $56.82 \pm 0.1 \mathrm{mN} / \mathrm{m}$, implying the presence of surface active impurities in the solution. Using pendant drop shape analysis, however, we found the surface tension of our solution to be $70.42 \pm 0.8 \mathrm{mN} / \mathrm{m}$ for the first 10 min, after which time the it slowly decreased to $\sim 57 \mathrm{mN} / \mathrm{m}$ over a span of $35 \mathrm{~min}$. The different results from these two techniques are likely due to the fact that the sessile bubble method incorporates a relatively large volume of solution with a relatively small surface area, allowing significant amounts of impurities to build up at the bubble interface. The pendant drop method, by contrast, involves a relatively small volume and relatively large surface area, and we therefore observed significant impurities diffusing to the surface only after long times. In our liquid jet experiments, we observe much smaller droplets with even higher surface area to volume ratios for times of $<1$ ms after formation. This implies that significant impurities are not likely to be present at the surfaces of the droplets during the time scale of our experiments. Moreover, even if some surface active impurities are present in our experiments, we do not expect our results to be sensitive to them. Numerous studies have shown that surface active species only affect the evaporation kinetics of water when there are relatively large amounts present (at least sufficient for a full monolayer) and typically 
only for larger surfactant molecules. ${ }^{36-40}$ At the concentrations expected in our study, we believe that any effects from such impurities are significantly smaller than the precision of our derived temperatures.

It seems likely, then, that the reduced $\gamma_{\mathrm{e}}$ is due to the surface enhancement of the perchlorate ion, but the exact nature of the kinetic effect is not immediately clear. While experimental studies of sodium perchlorate solutions have shown evidence for a strong surface partitioning of the perchlorate ion, they were unable to provide quantitative information about the expected surface concentration relative to that of the bulk. ${ }^{18,23}$ If we assume a surface concentration of $4 \mathrm{M}$, there should be one perchlorate ion for every 11.24 water molecules. If each water molecule at the air-water interface is assumed to have three hydrogen bonds to other species in the liquid, then 1 in every 3.74 water molecules, or $26.7 \%$, can be expected to be directly interacting with a perchlorate ion. Theoretical studies of hydration of the perchlorate ion show a large hydration radius, suggesting that the perchlorate ion forms relatively weak hydrogen bonds with water molecules. ${ }^{41,42}$ This is supported by attenuated total reflectance-Fourier transform infrared (FTIRATR) measurements, which indicate weaker interactions between water molecules and perchlorate ions than for water molecules interacting with other water molecules. ${ }^{43}$ Ultrafast measurements of solvated perchlorate ions, however, indicate that while the interactions between the ions and water molecules are weak, the orientational correlation time is significantly slowed for water molecules within the perchlorate solvation shell. ${ }^{44,45}$ Our own transition state theory (TST) model of water evaporation suggested that the evaporation kinetics are primarily influenced by the hindered translational and librational motions at the liquid surface, although the specific mechanism could not be determined. ${ }^{46}$ If perchlorate ions hinder the librational motions of surface water molecules, they may slow or completely inhibit evaporation for these molecules, leading to a lower value for $\gamma_{\mathrm{e}}$ relative to pure water, since fewer evaporation attempts would succeed.

The results of the present study do show, however, that inorganic solutes can indeed affect the evaporation kinetics of liquid water. Further studies on other inorganic solutes will likely prove interesting, although the small change observed in the present study suggests that ion effects are not necessarily strong and may be undetectable in many cases, even with high precision technique. Even so, studies of other surface active ions, such as thiocyanate, will help determine whether surface activity is the determining factor for kinetic effects on evaporation or if other specific ion effects are involved. Additionally, studies on acidic and basic solutions could prove insightful, as there is considerable debate over the role played by the hydronium and hydroxide ions at the air-water interface. ${ }^{47-49}$ Effects of hydronium on evaporation would also be pertinent to the atmosphere, where low $\mathrm{pH}$ is common in liquid aerosol. It seems likely, however, that the largest determinant for vapor-liquid exchange kinetics in the atmosphere is the presence of surface active organic species on droplets. ${ }^{13,14}$ New experimental techniques are needed to examine the effects of such coatings on evaporation with precision.

\section{Conclusions}

Using liquid microjets in a free evaporation regime, we have measured the evaporation coefficient of $4 \mathrm{M}$ sodium perchlorate solution to be $0.48 \pm 0.02$, roughly $25 \%$ smaller than our pure water value of $0.62 \pm 0.09 .{ }^{3}$ No such change was observed in our previous study of ammonium sulfate solution. ${ }^{15}$ The smaller evaporation coefficient is likely due to the expected surface enhancement of the perchlorate ion, allowing direct interactions between perchlorate and evaporating water molecules. The perchlorate ion has been shown to affect the orientational correlation time for water molecules within its solvation shell, ${ }^{44,45}$ suggesting that the ion may interfere with the librational motions of surface water molecules, leading to an inhibition of evaporation. Further theoretical work is needed to examine this possibility. While the perchlorate ion itself is not relevant in the atmosphere, its effects on evaporation suggest that certain inorganic solutes can alter the evaporation and condensation kinetics of water, especially if the solutes are expected to be enhanced in concentration at the air-water interface.

Acknowledgment. The authors thank Dr. Tatiana Svitova and Professor Clayton Radke for assistance with the surface tension measurements. This work was supported by National Science Foundation Grant ATM 0639847 and the Director, Office of Science, Office of Basic Energy Sciences, of the U.S. Department of Energy under Contract DE-AC02-05CH11231.

\section{References and Notes}

(1) Forster, P. V.; Ramaswamy, P.; Artaxo, T.; Berntsen, R.; Betts, D. W.; Fahey, J.; Haywood, J.; Lean, D. C.; Lowe, G.; Myhre, J.; Nganga, R.; Prinn, G.; Raga, M. S. a. R. V. D. Changes in Atmospheric Constituents and in Radiative Forcing. In Climate Change 2007: The Physical Science Basis. Contribution of Working Group I to the Fourth Assessment Report of the Intergovernmental Panel on Climate Change; Solomon, S., Qin, D., Manning, M., Chen, Z., Marquis, M., Averyt, K. B., Tignor, M., Miller, H. L., Eds.; Cambridge University Press: Cambridge, 2007.

(2) Drisdell, W. S.; Cappa, C. D.; Smith, J. D.; Saykally, R. J.; Cohen, R. C. Atmos. Chem. Phys. 2008, 8, 6699.

(3) Smith, J. D.; Cappa, C. D.; Drisdell, W. S.; Cohen, R. C.; Saykally, R. J. J. Am. Chem. Soc. 2006, 128, 12892.

(4) Eames, I. W.; Marr, N. J.; Sabir, H. Int. J. Heat Mass Transfer 1997, 40, 2963.

(5) Marek, R.; Straub, J. Int. J. Heat Mass Transfer 2001, 44, 39.

(6) Li, Y. Q.; Davidovits, P.; Shi, Q.; Jayne, J. T.; Kolb, C. E.; Worsnop, D. R. J. Phys. Chem. A 2001, 105, 10627.

(7) Davidovits, P.; Kolb, C. E.; Williams, L. R.; Jayne, J. T.; Worsnop, D. R. Chem. Rev. 2006, 106, 1323.

(8) Winkler, P. M.; Vrtala, A.; Rudolf, R.; Wagner, P. E.; Riipinen, I.; Vesala, T.; Lehtinen, K. E. J.; Viisanen, Y.; Kulmala, M. J. Geophys. Res. [Atmos.] 2006, 111,

(9) Zientara, M.; Jakubczyk, D.; Kolwas, K.; Kolwas, M. J. Phys. Chem. A 2008, 112, 5152 .

(10) Cappa, C. D.; Drisdell, W. S.; Smith, J. D.; Saykally, R. J.; Cohen, R. C. J. Phys. Chem. B 2005, 109, 24391.

(11) Fukuta, N.; Myers, M. N. J. Atmos. Sci. 2007, 64, 955.

(12) Chuang, P. Y.; Charlson, R. J.; Seinfeld, J. H. Nature 1997, 390, 594.

(13) Ruehl, C. R.; Chuang, P. Y.; Nenes, A. Atmos. Chem. Phys. 2008, $8,1043$.

(14) Shantz, N. C.; Chang, R. Y. W.; Slowik, J. G.; Vlasenko, A.; Abbatt, J. P. D.; Leaitch, W. R. Atmos. Chem. Phys. 2010, 10, 299.

(15) Drisdell, W. S.; Saykally, R. J.; Cohen, R. C. Proc. Natl. Acad. Sci. U.S.A. 2009, 106, 18897.

(16) Zhang, Q.; Jimenez, J. L.; Canagaratna, M. R.; Allan, J. D.; Coe, H.; Ulbrich, I.; Alfarra, M. R.; Takami, A.; Middlebrook, A. M.; Sun, Y. L.; Dzepina, K.; Dunlea, E.; Docherty, K.; DeCarlo, P. F.; Salcedo, D.; Onasch, T.; Jayne, J. T.; Miyoshi, T.; Shimono, A.; Hatakeyama, S.; Takegawa, N.; Kondo, Y.; Schneider, J.; Drewnick, F.; Borrmann, S.; Weimer, S.; Demerjian, K.; Williams, P.; Bower, K.; Bahreini, R.; Cottrell, L.; Griffin, R. J.; Rautiainen, J.; Sun, J. Y.; Zhang, Y. M.; Worsnop, D. R. Geophys. Rev. Lett. 2007, 34.

(17) Gopalakrishnan, S.; Jungwirth, P.; Tobias, D. J.; Allen, H. C. J. Phys. Chem. B 2005, 109, 8861.

(18) Pegram, L. M.; Record, M. T. J. Phys. Chem. B 2007, 111, 5411.

(19) Gopalakrishnan, S.; Liu, D. F.; Allen, H. C.; Kuo, M.; Shultz, M. J. Chem. Rev. 2006, 106, 1155.

(20) Thomas, J. L.; Roeselova, M.; Dang, L. X.; Tobias, D. J. J. Phys. Chem. A 2007, 111, 3091.

(21) Kido Soule, M. C.; Blower, P. G.; Richmond, G. L. J. Phys. Chem. B 2007, 111, 13703. 
(22) Otten, D. E.; Petersen, P. B.; Saykally, R. J. Chem. Phys. Lett. 2007, 449, 261.

(23) Cheng, J.; Vecitis, C. D.; Hoffmann, M. R.; Colussi, A. J. J. Phys. Chem. B 2006, 110, 25598.

(24) Walrafen, G. E. J. Chem. Phys. 1970, 52, 4176.

(25) Murphy, D. M.; Koop, T. Q. J. R. Meteorol. Soc. 2005, 131, 1539.

(26) Djogic, R.; Branica, M. Anal. Chim. Acta 1993, 281, 291.

(27) Fontan, C. F.; Chirife, J. J. Food Technol. 1981, 16, 21.

(28) Chelf, J. H.; Martin, S. T. Geophys. Rev. Lett. 1999, 26, 2391.

(29) Chirife, J.; Resnik, S. L. J. Food Sci. 1984, 49, 1486.

(30) Roth, K.; Wolf, U.; Wolf, G. CALPHAD: Comput. Coupling Phase Diagrams Thermochem. 1997, 21, 475.

(31) Riedel, L. Chem. Ing. Tech. 1951, 23, 59.

(32) Hare, D. E.; Sorensen, C. M. J. Chem. Phys. 1987, 87, 4840.

(33) Scheffler, K. IAPWS, 2008.

(34) Hiquily, N.; Clifton, M. J. J. Chem. Eng. Data 1984, 29, 371.

(35) Weissenborn, P. K.; Pugh, R. J. J. Colloid Interface Sci. 1996, 184, 550.

(36) Lunkenheimer, K.; Zembala, M. J. Colloid Interface Sci. 1997, 188, 363.

(37) Rusdi, M.; Moroi, Y. J. Colloid Interface Sci. 2004, 272, 472.
(38) Rusdi, M.; Moroi, Y.; Nakahara, H.; Shibata, O. Langmuir 2005, 21,7308 .

(39) Lawrence, J. R.; Glass, S. V.; Nathanson, G. M. J. Phys. Chem. A $\mathbf{2 0 0 5}, 109,7449$.

(40) Buajarern, J.; Mitchem, L.; Reid, J. P. J. Phys. Chem. A 2007, 111, 11852 .

(41) Conway, B. E.; Ayranci, E. J. Solution Chem. 1999, 28, 163.

(42) Li, C. X.; Lee, H. Chem. Eng. Sci. 2000, 55, 655.

(43) Wei, Z. F.; Zhang, Y. H.; Zhao, L. J.; Liu, J. H.; Li, X. H. J. Phys. Chem. A 2005, 109, 1337.

(44) Omta, A. W.; Kropman, M. F.; Woutersen, S.; Bakker, H. J. J. Chem. Phys. 2003, 119, 12457.

(45) Omta, A. W.; Kropman, M. F.; Woutersen, S.; Bakker, H. J. Science 2003, 301, 347.

(46) Cappa, C. D.; Smith, J. D.; Drisdell, W. S.; Saykally, R. J.; Cohen, R. C. J. Phys. Chem. C 2007, 111, 7011.

(47) Petersen, P. B.; Saykally, R. J. J. Phys. Chem. B 2005, 109, 7976.

(48) Petersen, P. B.; Saykally, R. J. Chem. Phys. Lett. 2008, 458, 255.

(49) Gray-Weale, A.; Beattie, J. K. Phys. Chem. Chem. Phys. 2009, 11, 10994.

JP101726X 